\title{
RARE EVENT SIMULATION AND SPLITTING FOR DISCONTINUOUS RANDOM VARIABLES *
}

\author{
Clément Walter ${ }^{1,2}$
}

\begin{abstract}
Multilevel Splitting methods, also called Sequential Monte-Carlo or Subset Simulation, are widely used methods for estimating extreme probabilities of the form $\mathrm{P}[S(\mathbf{U})>q]$ where $S$ is a deterministic real-valued function and $\mathbf{U}$ can be a random finite- or infinite-dimensional vector. Very often, $X:=S(\mathbf{U})$ is supposed to be a continuous random variable and a lot of theoretical results on the statistical behaviour of the estimator are now derived with this hypothesis. However, as soon as some threshold effect appears in $S$ and/or $\mathbf{U}$ is discrete or mixed discrete/continuous this assumption does not hold any more and the estimator is not consistent. In this paper, we study the impact of discontinuities in the $c d f$ of $X$ and present three unbiased corrected estimators to handle them. These estimators do not require to know in advance if $X$ is actually discontinuous or not and become all equal if $X$ is continuous. Especially, one of them has the same statistical properties in any case. Efficiency is shown on a 2-D diffusive process as well as on the Boolean SATisfiability problem (SAT).
\end{abstract}

Mathematics Subject Classification. 65C05, 65C60, 62L12, 62N02.

Received July 3, 2015. Revised November 2, 2015.

\section{INTRODUCTION}

In the context of reliability analysis, one is often concerned with the estimation of extreme quantile or probability. Indeed one goal of uncertainty quantification is to estimate the probability of failure of a given system and inversely, quantile estimation helps defining guidelines to insure a good behaviour of the system with a given probability of failure. Usually, the system is considered as a blackbox (often a complex numerical code) which returns a real value defining its state. According to this output, it is then considered as working properly or not.

Formally, the problem can be written as follows: let $\mathbf{U}$ be a random finite- or infinite-dimensional vector with known distribution $\mu^{U}$ and $S$ a performance function (the computer code for instance), one seeks for estimating $p$ given $q$ (or $q$ given $p$ ) such that $p=\mathrm{P}[S(\mathbf{U})>q]$. The main difficulties arise from the fact that

1) the sought probability or quantile is extreme, say $p<10^{-5}$ and

2) the computer code is very time consuming.

Keywords and phrases. Rare event simulation, multilevel splitting, RESTART, sequential Monte Carlo, extreme event estimation, counting, Last Particle Algorithm.

* This work was partially supported by ANR project Chorus.

1 CEA, DAM, DIF, 91297 Arpajon, France. walter@math.univ-paris-diderot.fr

2 Laboratoire de Probabilités et Modèles Aléatoires, Université Paris Diderot, 75205 Paris cedex 05, France. 
All together, these two characteristics prevent from using a naive Monte-Carlo estimator:

$$
\widehat{p}_{\mathrm{MC}}=\frac{1}{N} \sum_{i=1}^{N} S\left(\mathbf{U}_{i}\right)
$$

with $\left(\mathbf{U}_{i}\right)_{i}$ an iid sample with distribution $\mu^{U}$ because $\mathrm{CV}\left[\widehat{p}_{\mathrm{MC}}\right]^{2} \approx(N p)^{-1}$, which means that one would require $N=10^{2} / p$ to get a coefficient of variation of $10 \%$.

In this scope other statistics have been defined to get low variance estimators. Among them the Multilevel Splitting method (MS) $[15,16,20,23]$ rewrites the sought probability using the Bayes's rule and a finite sequence of increasing thresholds $\left(q_{i}\right)_{i=0}^{m}$ such that $q_{0}=-\infty$ and $q_{m}=q$ :

$$
\mathrm{P}[S(\mathbf{U})>q]=\mathrm{P}\left[S(\mathbf{U})>q_{m} \mid S(\mathbf{U})>q_{m-1}\right] \times \ldots \times \mathrm{P}\left[S(\mathbf{U})>q_{2} \mid S(\mathbf{U})>q_{1}\right] \mathrm{P}\left[S(\mathbf{U})>q_{1}\right] .
$$

From equation (1.2) the goal is then to estimate separately each conditional probability with a crude Monte Carlo. Hence, the sequence $\left(q_{j}\right)_{j}$ has to be chosen such that each probability is not too small to make its estimation with crude Monte-Carlo feasible $[2,25]$. The variance of the estimator depends on the choice of this sequence and especially it is known that the conditional probabilities should be all equal to minimize it [11]. A typical MS algorithm works as follows:

(1) Sample a Monte-Carlo population $\left(\mathbf{U}_{i}\right)_{i}$ of size $N ; j=0$.

(2) Estimate the conditional probability $\mathrm{P}\left[S(\mathbf{U})>q_{j+1} \mid S(\mathbf{U})>q_{j}\right]$.

(3) Resample the $\mathbf{U}_{i}$ such that $S\left(\mathbf{U}_{i}\right) \leq q_{j+1}$ conditionally to be greater than $q_{j+1}$ (the other ones do not change).

(4) $j \leftarrow j+1$ and repeat until $j=m$.

When it is not possible to infer such a sequence $\left(q_{j}\right)_{j}$, it is usual to define it on-the-fly while the algorithm is running and this is known as adaptive multilevel splitting (AMS). The sequence is built either by fixing the conditional probabilities to be all equal to some given value $p_{0} \in(0.1,0.5)$ [2], or by using the $k$ th order statistics and so to estimate the corresponding probability by $1-k / N$. In the first case, $\left(q_{j}\right)_{j}$ is then a sequence of estimated quantiles with crude Monte Carlo while in the second case it is a sequence of arbitrarily chosen thresholds and the corresponding probabilities are estimated with crude Monte Carlo. Hence the first option leads to a bias in the final estimator $[9,11]$ while the latter produces an unbiased estimator for any $k[7]$. The link with Interacting Particle Systems [13] allowed for deriving a lot of theoretical results on the optimal number of subsets, the statistical behaviour of the estimator and the impact of the adaptive method $[3,8]$. Furthermore, the special case of the Last Particle Algorithm (AMS with $k=1$ ) has gained a lot of attention recently. It has the smallest variance amongst all AMS [7]; especially [17,28] showed that the random number of iterations of the algorithm follows a Poisson's law when $X$ is continuous. Moreover [31], following [19], brought an original insight in terms of a random walk of the real-valued random variable $X=S(\mathbf{U})$ linked with a Poisson's Process with parameter 1. Indeed the estimator is found to be the Minimal Variance Unbiased Estimator (MVUE) of the exponential of a parameter of a Poisson's law with $N$ iid. realisations of such random variables. As a consequence it turns it into the optimal (minimal variance) parallel Multilevel Splitting estimator [30,32].

However, all these results assume that the $c d f$ of $X$ is continuous and little is known about the impact of using such strategies if this assumption does not hold. This case can happen if for example there is some threshold effect in $S$ and/or if $\mathbf{U}$ is discrete or mixed discrete/continuous [10,24-26]. Recently, [28] showed that in the case of the Last Particle Algorithm the random number of iterations is indeed a mixture of independent Poisson and negative binomial laws while in the continuous case, it is only a Poisson's law. Unfortunately he could not derive a general unbiased estimator from this result ([28], Thms. 4 and 5). The main problem comes from the fact that the equality $\forall x \in \mathbb{R}, \mathrm{P}[X>x]=\mathrm{P}[X \geq x]$ does not hold any more. [24] already noticed that one should pay attention to the fact that the root $q_{i}$ of $\mathrm{P}\left[S(\mathbf{U}) \geq q_{i} \mid S(\mathbf{U}) \geq q_{i-1}\right]=p_{0}$ may not be unique ([24], Rem. 6.1, [5], Rem. 2.6). He then derived some guidelines for an appropriate adaptive choice of the $\left(q_{i}\right)_{i}$ in 
this case but concludes that the algorithm can eventually fail to estimate the sought probability (it stops at an intermediate level (see [24], Rem. 6.3) or returns 0 [1]). Finally, [10] suggested to use for the SAT problem an auxiliary continuous random variable $Y$ such that $\mathrm{P}[Y>q]=\mathrm{P}[S(\mathbf{U})>q]$ and showed practical improvement. This strategy is also used by [19] for the Ising model but these transformations are always case specific. [29] also mentions this issue and proposes to add a uniform random variable on a tiny interval but one lacks of justifications and clear guidelines and consequences.

Following the random walk framework from [31] the goal of this paper is to fill this gap by providing both the distribution of the number of iterations and the MVUE estimator in each case: the one with strict inequality and the one with non-strict inequality. Indeed the decomposition of equation (1.2) suggests these two possible definitions for a Multilevel Splitting method. While there are the same with probability 1 if $X$ is continuous, they may differ if $X$ is not. Especially recent results from [12] derive an unbiased estimator for the strict inequality case in the usual AMS framework. However the distributions of the estimators are less simple than in the continuous case. In this scope we also suggest a third estimator based on the random walk with non-strict inequality which has the same statistical properties as in the continuous case. Practically speaking it is not necessary to know in advance if $X$ is actually continuous or not and in this latter case, the three estimators become the same.

The paper is organized as follows: first we recall the theoretical results for continuous random variables. Then we show how discontinuities alter them and we give corresponding corrected estimators. Finally, we apply this modified version to a dynamic test case from [28], it is the probability of a Markov process reaching a given set $B$ before going to another set $A$, and to a counting problem, namely the Boolean SATisfiability problem [18].

\section{RARE EVENT SIMULATION FOR DISCONTINUOUS RANDOM VARIABLES}

\subsection{The increasing random walk}

In this section, we recall common results from $[17,19,28]$ in the framework of [31]. Let us consider $X=$ $S(\mathbf{U}) \in \mathbb{R}$ a real-valued random variable with distribution $\mu^{X}$ where $S$ is a deterministic function (for instance the output of a computer code) and $\mathbf{U}$ a random finite- or infinite-dimensional vector with known distribution $\mu^{U}$. In this section we assume that the $c d f F_{X}$ of $X$ is continuous.

Definition 2.1 (increasing random walk). Let $X$ be a real-valued random variable with continuous $c d f F_{X}$, $X_{0}=-\infty$ and define the Markov's sequence $\left(X_{n}\right)_{n}$ such that:

$$
\forall n \in \mathbb{N}, \mathrm{P}\left[X_{n+1} \in A \mid X_{0}, \ldots, X_{n}\right]=\frac{\mathrm{P}\left[X \in A \cap\left(X_{n},+\infty\right)\right]}{\mathrm{P}\left[X \in\left(X_{n},+\infty\right)\right]} .
$$

In other words $\left(X_{n}\right)_{n}$ is an increasing sequence where each element is randomly generated conditionally greater than the previous one: $X_{n+1} \sim \mu^{X}\left(\cdot \mid X>X_{n}\right)$. Assuming that $F_{X}$ is continuous, the associated sequence $\left(T_{n}\right)_{n}$ such that $T_{n}=-\log \mathrm{P}\left[X>X_{n}\right]$ is distributed as the arrival times of a Poisson's Process with parameter 1.

Remark 2.2. Since $X$ is continuous, the random walk can alternatively be defined with non-strict inequality: $X_{n+1} \sim \mu^{X}\left(\cdot \mid X \geq X_{n}\right)$ without changing this result.

In the sequel, we then consider for all $x \in \mathbb{R}$ the associated numbers $p_{x}=\mathrm{P}[X>x]$ and $t_{x}=-\log p_{x}$. Hence, for all $x \in \mathbb{R}$, the counting random variable of the number of events before $x: M_{x}=\operatorname{card}\left\{n \in \mathbb{N} \mid X_{n} \leq x\right\}$ follows a Poisson's law with parameter $t_{x}=-\log p_{x}$. From this result one can build an estimator for $p_{x}$. Indeed, simulating $N$ iid. Poisson random variables with the same parameter $t_{x}:\left(M_{x}^{i}\right)_{i=1}^{N}$, one seeks for estimating $\mathrm{e}^{-t_{x}}$. In this context the Lehmann-Scheffé's theorem insures that the Minimal-Variance Unbiased Estimator (MVUE) is:

$$
\widehat{p}_{x}=\left(1-\frac{1}{N}\right)^{\sum_{i=1}^{N} M_{x}^{i}} .
$$




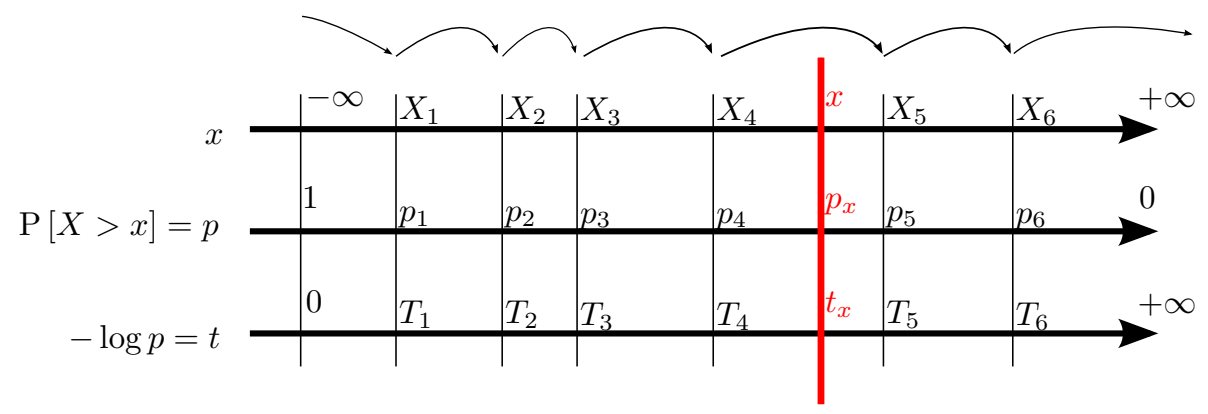

Figure 1. The increasing random walk and the associated Poisson's process.

Proposition 2.3 (Statistical properties of the probability estimator).

$$
\begin{gathered}
\widehat{p}_{x} \underset{N \rightarrow \infty}{\stackrel{a . s}{\longrightarrow}} p_{x} \\
\operatorname{var}\left[\widehat{p}_{x}\right]=p_{x}^{2}\left(p_{x}^{-1 / N}-1\right) .
\end{gathered}
$$

This estimator exhibits a logarithmic efficiency and asymptotically achieves the Cramer-Rao bound $-p_{x}^{2} \log p_{x} / N$.

Remark 2.4. The Last Particle Algorithm $[17,28]$ is only one possible implementation of this estimator; especially [30] studied its parallelisation and showed that it generates a marked Poisson's process with parameter $N$. On the other hand, [19] presented the increasing random walk linked with a Poisson's process with parameter 1 but focused on $\log p_{x}$ instead of $p_{x}$.

Remark 2.5. When generating $M_{x}$ a counting random variable at given state $x \in \mathbb{R}$, one indeed generates $M_{x_{0}}$ for all $x_{0} \leq x$. This gives a Glivenko-Cantelli like results:

$$
\forall x_{0} \leq x, F_{N}\left(x_{0}\right)=1-\left(1-\frac{1}{N}\right)^{M_{x_{0}}} \underset{N \rightarrow \infty}{\stackrel{a . s .}{\longrightarrow}} F_{X}\left(x_{0}\right)
$$

with $M_{x_{0}}$ the sum of $N$ iid. counting random variables at state $x_{0}$.

With several realisations of the increasing random walk it is also possible to define parallel quantile and mean estimators for continuous random variables. The interested reader is referred to $[17,30]$ for quantile estimation and [31] for mean estimation.

\subsection{Extension of the random walk for discontinuous random variables}

We now intend to extend the definition of the random walk of Section 2.1 to the case where $X$ is not necessarily continuous - or potentially discrete. Especially, while for all $x \in \mathbb{R}, \mathrm{P}[X>x]=\mathrm{P}[X \geq x]$ when $F_{X}$ is continuous, there are now two alternative definitions for the increasing random walk (see Def. 2.1), precisely the one with strict inequality and the one with non-strict inequality (see Rem. 2.2). In this latter case [28] showed that the counting random variable at a given state $x$ follows indeed a mixture of a Poisson's law and independent Geometric laws with parameters depending on the atoms of $X$. This result prevented him from getting an unbiased estimator for $p_{x}$. Instead he focused on the special case with only one jump point $x_{0}$ reached at the first iteration of the random walk. In this context he proposed to estimate the atom with a crude Monte Carlo on the first iid. sampling and then to consider the continuous random variable $X$ with the conditional distribution given $X>x_{0}$, that is the distribution with $c d f \mathbb{1}_{x>x_{0}}\left(F_{X}(x)-F_{X}\left(x_{0}\right)\right) /\left(1-F_{X}\left(x_{0}\right)\right)$ ([28], Thm. 5). 
In this paper we go one step beyond his work by providing a general MVUE for the non-strict inequality random walk as well as for the strict inequality random walk. We also introduce a method for getting an estimator with the same properties as above (Prop. 2.3) even if the random variable is discontinuous, but without any intrusive nor case specific trick as in [10] or [19].

From now on, we assume that $X$ is a real-valued random variable, continuous or not, and we denote by $D$ the set of its atoms. According to Froda's theorem [14] it is countable. As in Section 2.1 we consider for all $x \in \mathbb{R}$ the counting random variable $M_{x}=\operatorname{card}\left\{n \in \mathbb{N} \mid X_{n} \leq x\right\}$. The next two propositions aim at giving the law of $M_{x}$ in the strict and non-strict cases. In both Propositions 2.6 and 2.7, we consider $x \in \mathbb{R} \mid p_{x}=\mathrm{P}[X>x]>0$, $D_{x}=D \cap(-\infty, x]$ and define: $\forall d \in D_{x}$ :

$$
\Delta_{d}=\frac{\mathrm{P}[X>d]}{\mathrm{P}[X \geq d]}
$$

Proposition 2.6 (Law of the counting random variable for the non-strict random walk).

$$
M_{x} \sim \mathcal{P}\left(-\log \frac{p_{x}}{\prod_{d \in D_{x}} \Delta_{d}}\right)+\sum_{d \in D_{x}} \mathcal{G}\left(\Delta_{d}\right)
$$

with $\mathcal{G}$ a Geometric law counting the number of failures before success.

The distribution of $M_{x}$ is that of the sum of a Poisson random variable with parameter $-\log p_{x} / \prod \Delta_{d}$ and of Geometric random variables with parameters $\left(\Delta_{d}\right)_{d \in D_{x}}$; these random variables being independent. It is part of the proof given in Appendix that the distribution is well defined with finite mean and variance even when $\operatorname{card}\left(D_{x}\right)=\infty$. Also this result was already stated in [28] with a combinatorial analysis assuming that $\operatorname{card}(D)<\infty$. Indeed it can be understood using the renewal property of a Poisson's Process: the number of events corresponding to the continuous part, i.e. events $X_{n} \notin D$, follows a Poisson's law with parameter $-\log p_{x}-\sum\left(-\log \Delta_{d}\right)=-\log \left(p_{x} / \prod \Delta_{d}\right)$. On the other hand each jump point leads to a random number of iterations following a Geometric law with probability of success $\mathrm{P}[X>d] / \mathrm{P}[X \geq d]=\Delta_{d}$.

Proposition 2.7 (Law of the counting random variable for the strict random walk).

$$
M_{x} \sim \mathcal{P}\left(-\log \frac{p_{x}}{\prod_{d \in D_{x}} \Delta_{d}}\right)+\sum_{d \in D_{x}} \mathcal{B}\left(1-\Delta_{d}\right)
$$

with $\mathcal{B}$ a Bernoulli distribution.

Hence the strict inequality random walk replaces the Geometric distribution by a Bernoulli one. Since the Geometric law counts the number of failures while the Bernoulli one gives 1 in case of success, the parameter is the opposite. Furthermore, both equations (2.7) and (2.8) are equal when $D=\varnothing$, i.e. when $X$ is continuous. In this latter case, one finds back the pure Poisson's distribution $M_{x} \sim \mathcal{P}\left(-\log p_{x}\right)$.

\subsection{Probability estimators}

As noticed by [28] the formulas (2.7) and (2.8) are not very useful to derive an unbiased probability estimator. However we do not generate only iid. copies of the counting random variables but the random walks themselves. Hence if one can afford storing all the states of each random walk, then it is possible to build a MVUE in both cases. 
Lemma 2.8 (MVUE for a Geometric distribution). Let $G \sim \mathcal{G}(p)$ be a Geometric random variable counting the number of failures before success with probability of success $p$ and $\left(G_{i}\right)_{i=1}^{N} N$ iid. copies of $G$, then the minimal variance unbiased estimator for $p$ is:

$$
\widehat{p}=\frac{N-1}{N-1+\sum_{i=1}^{N} G_{i}}
$$

Lemma 2.9 (MVUE for a Bernoulli's distribution). Let $B \sim \mathcal{B}(1-p)$ be a Bernoulli random variable with probability of failure $p$ and $\left(B_{i}\right)_{i=1}^{N} N$ iid. copies of $B$, then the minimal variance unbiased estimator for $p$ is:

$$
\widehat{p}=1-\frac{\sum_{i=1}^{N} B_{i}}{N}
$$

Definition 2.10 (Run-length encoding). Let $\mathbf{v}=\left(v_{1}, \ldots, v_{m}\right) \in \mathbb{R}^{m}, m \geq 1$ be a vector such that $\forall i \in$ $\llbracket 1, m-1 \rrbracket, v_{i} \leq v_{i+1}$. We call the run-length encoding of $\mathbf{v}$ the vector $\mathbf{r}$ of the lengths of runs of equal values in $\mathbf{v}$.

In other words, the run-length encoding counts for any non decreasing sequence the number of times each value is repeated: for example if $\mathbf{v}=(0.5,2.1,2.1,2.1, \pi)$ then $\mathbf{r}=(1,3,1)$. Especially, if $X$ is continuous the RLE of the states of a realisation of the increasing random walk $\left(X_{1}, \ldots, X_{m}\right)$, is $\mathbf{r}=(1, \ldots, 1) \in \mathbb{R}^{m}$ with probability 1 while on the contrary discontinuities will produce repeated values with non-zero probability. More precisely, the number of times each value is repeated corresponds to the number of failures while sampling above a threshold. With this consideration we are now in position to define the probability estimators.

In the sequel we assume that for a given $x \in \mathbb{R} \mid \mathrm{P}[X>x]>0,\left(X_{i}\right)_{i=1}^{M_{x}}$ is the merged and sorted sequence of the states of $N$ (non-)strict inequality random walks generated until state $x ; M_{x}=\sum_{i=1}^{N} M_{x}^{i}$ is the sum of the counting random variables of each random walk, $\mathbf{r}$ is the RLE of $\left(X_{1}, \ldots, X_{M_{x}}\right)$, and $l$ is its length.

Proposition 2.11. The MVUE for the non-strict inequality random walk is:

$$
\widehat{p}_{x}=\prod_{i=1}^{l} \frac{N-1}{N-1+r_{i}} .
$$

It verifies:

$$
p_{x}^{2}\left(p_{\text {pois }}^{-1 / N}-1\right) \leq \operatorname{var}\left[\widehat{p}_{x}\right] \leq p_{x}^{2}\left(p_{\text {pois }}^{-1 / N}\left(\frac{N-1}{N-2}\right)^{\# D_{x}}-1\right)
$$

with $p_{\text {pois }}=\frac{p_{x}}{\prod_{d \in D_{x}} \Delta_{d}}$ and \# $D_{x}=\operatorname{card}\left(D_{x}\right)$.

It is interesting to notice here that in a case of a discrete random variable, $p_{\text {pois }}=1$ and the bounds on the variance become:

$$
0 \leq \frac{\operatorname{var}\left[\widehat{p}_{x}\right]}{p_{x}^{2}} \leq\left(\frac{N-1}{N-2}\right)^{\# D_{x}}-1
$$

It means that the coefficient of variation is bounded by a quantity which does not depend on the size but only on the number of jumps. Since this quantity is likely to be known with good confidence and does not vary much with $\# D_{x}$, this can provide a robust upper bound for the coefficient of variation. 
Proposition 2.12. The MVUE for the strict inequality random walk is:

$$
\widehat{p}_{x}=\prod_{i=1}^{l}\left(1-\frac{r_{i}}{N}\right) .
$$

It verifies:

$$
\operatorname{var}\left[\widehat{p}_{x}\right]=p_{x}^{2}\left(p_{x}^{-1 / N} \prod_{d \in D_{x}} g\left(\Delta_{d}, N\right)-1\right)
$$

with $g:(\Delta, N) \mapsto \frac{\Delta(N-1)+1}{N \Delta^{1-1 / N}}$.

On the one hand we have been able to define minimal variance unbiased estimators for both the strict and non-strict random walks. They become equal when the random variable is continuous and in this case one finds back the estimator defined in Section 2.1. Especially the variance increase due to discontinuities in the distribution of $X$ is clearly visible in equation (2.15) as $g>1$ if $\Delta<1$. On the other hand their distributions are not easy to characterise; in particular we could not derive any practical literal expression of the variance in the non-strict case. In this context we suggest to consider an auxiliary continuous random variable which involves an independent uniform random variable. This transformation is general and does not require any other knowledge on the problem.

Indeed when generating a Geometric random variable with iid. Bernoulli trials, one can also consider the random variable $Y=B+U$ with $B \sim \mathcal{B}(p)$ the Bernoulli random variable with probability of success $p$ and $U \sim \mathcal{U}[0,1]$ an independent Uniform random variable on the interval $[0,1]$. Figure 2 plots the $c d f$ of $B$ and $Y$. Furthermore, $\forall y \in[0,2],\{Y>y\}=\{\{B \geq\lfloor y\rfloor\} \cap\{U>Y-\lfloor y\rfloor\}\}$. Practically speaking, this means that the generation of the geometric random variable can be seen as a basic Acceptance-Rejection scheme used to generate the increasing random walk on $Y$ until state 1 : for each generated $B$, sample also $U \sim \mathcal{U}[0,1]$ and accept the transition for $Y$ if $U>y-\lfloor y\rfloor$. Eventually, considering the fact that $p=\mathrm{P}[B=1]=\mathrm{P}[Y \geq 1]=\mathrm{P}[Y>1]$, $p$ can also be estimated using the increasing random walk on the continuous random variable $Y$. To conclude, in addition to the MVUE of $p$ defined in Lemma 2.8 and at the cost of the generation of an independent uniform random variable, one also produces an estimator of the form of equation (2.2) with the same statistical properties. Embedding this in the generation of the non-strict inequality random walk gives then an estimator with the same properties as the one in the continuous case. Algorithm 1, Theorem 2.13 and Corollary 2.14 precise this point. In the sequel, we will refer to this estimator as the pure Poisson estimator.

Theorem 2.13. In Algorithm 1 , the random variable $M$ follows a Poisson's law with parameter $-\log \mathrm{P}[X>x]$.

Corollary 2.14. Let $N \geq 2$ and $M=\sum_{i=1}^{N} M_{i}$ the sum of $N$ iid. realisations of Algorithm 1, the estimator

$$
\widehat{p}_{x}=\left(1-\frac{1}{N}\right)^{M}
$$

has the same properties as in Proposition 2.3.

Finally the distinction between the strict and non-strict random walks lets define two different estimators for the probability of exceeding a threshold. Both are unbiased and become the same when $X$ is indeed continuous. However their distributions are not well-characterised. In this scope we have introduced a third estimator based on the non-strict random walk. With the addition of a while loop and a Uniform random variable, we have been able to produce an estimator which has always the same statistical properties, $X$ being continuous or not. This estimator is not optimal in terms of variance when $X$ is actually discontinuous but remains close to it when the jumps $\left(\Delta_{d}\right)_{d}$ remain close to 1 : the MVUE of Lemma 2.8 has a squared coefficient of variation approximately equal to $(1-\Delta) / N$ while it is $-\log (\Delta) / N$ for the pure Poisson estimator. These results are illustrated in the next section. 


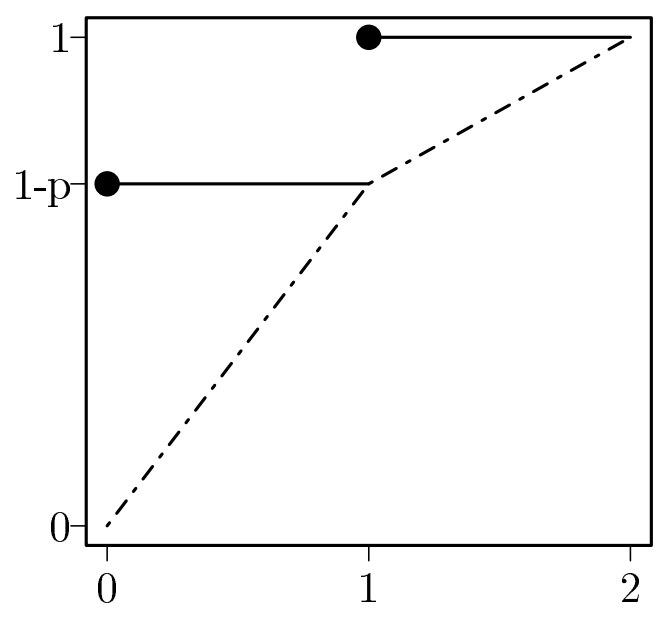

Figure 2. $c d f$ of a Bernoulli random variable $B \sim \mathcal{B}(p)$ (plain dark line) and its associated continuous random variable $Y=B+U$ (dark dashed line) with $U \sim \mathcal{U}[0,1]$.

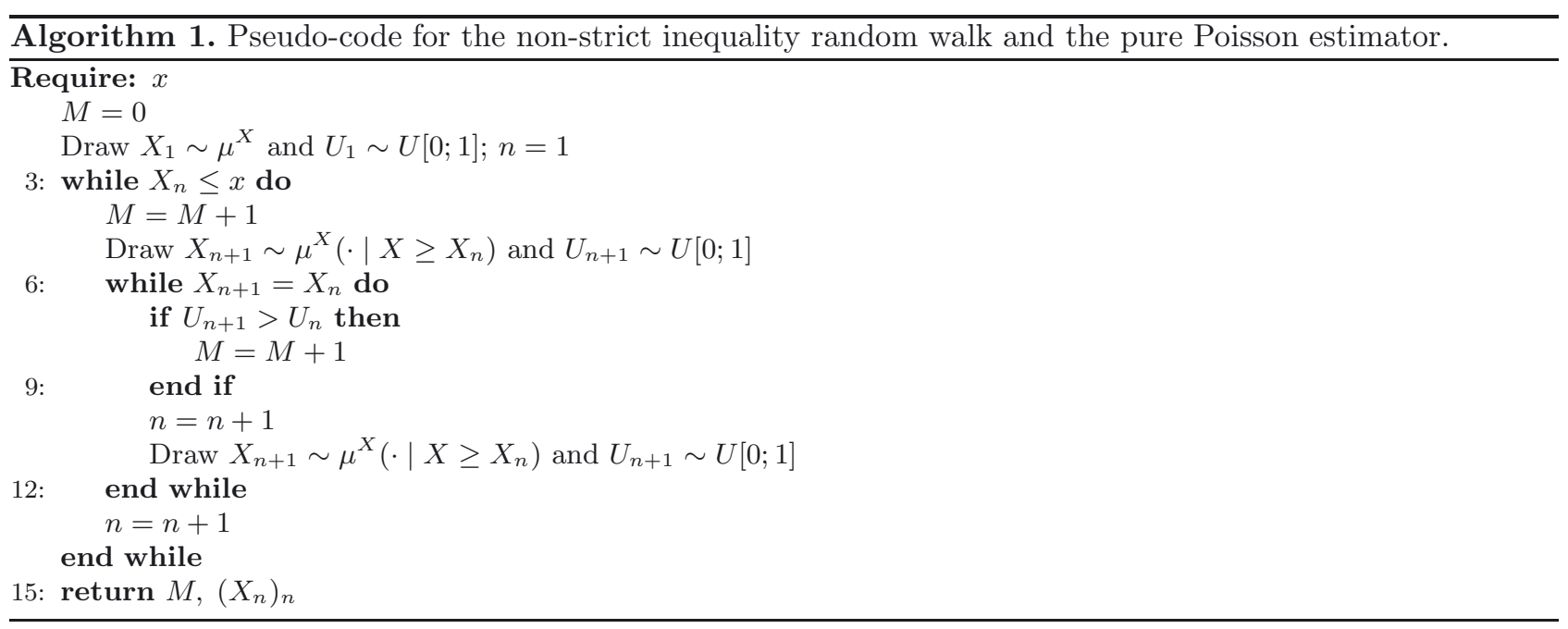

\section{EXAMPLES}

\subsection{Discontinuous random variable}

Problem setting

We consider here the example used by [28] to illustrate his results. It is a numerical study with a Euler's scheme of a diffusive process satisfying:

$$
\mathrm{d} \mathbf{U}_{t}=-\nabla V \mathrm{~d} t+\sqrt{\frac{2}{\beta}} \mathrm{d} \mathbf{W}_{t}, \mathbf{U}_{0}=\mathbf{u}_{0}
$$

where $\mathbf{W}_{t}$ is a Wiener's process, $\beta^{-1}$ is the temperature, and $V$ is a potential defined by:

$$
V\left(u_{1}, u_{2}\right)=-\left(\frac{u_{1}^{2}}{2}-\frac{u_{1}^{4}}{4}\right)-b\left(\frac{u_{2}^{2}}{2}-\frac{u_{2}^{4}}{4}\right)+\frac{a}{2} u_{1}^{2} u_{2}^{2},
$$


for some $a$ and $b$. The goal is to estimate the probability that the process enters a given set $B$ before another set $A$ from an initial state $\mathbf{u}_{0}$ : if $\tau_{C}$ is the stopping time defined by $\tau_{C}:=\inf \left\{t \geq 0 \mid \mathbf{U}_{t} \in C\right\}$, then one seeks for estimating:

$$
p=P_{\mathbf{u}_{0}}\left[\tau_{B}<\tau_{A}\right],
$$

where $P_{\mathbf{u}_{0}}$ is the distribution of $\left(\mathbf{U}_{t}\right)_{t}$ starting from $\mathbf{U}_{0}=\mathbf{u}_{0}$. As a function of $\mathbf{u}_{0}$ this quantity is known as the Committor. From a practical point of view, it is often intractable and a reaction coordinate is introduced to measure how far a trajectory is escaping from $A$ before returning to it: $\Phi: \mathbb{R}^{d} \rightarrow \mathbb{R}$ such that $A=\Phi^{-1}((-\infty, 0])$ and $B=\Phi^{-1}([1,+\infty))$. With these notations, a trajectory enters $B$ before returning to $A$ if and only if:

$$
X:=\sup _{t \in\left[0, \tau_{A}\right)} \Phi\left(\mathbf{U}_{t}\right) \geq 1
$$

$X$ is then the real-valued random variable of interest and the problem is indeed to estimate $\mathrm{P}[X \geq 1]$. With this notation, the theoretical results of Section 2 can be used directly.

\section{Conditional sampling}

This is the only requirement of the estimators defined in Section 2.3 but also the crucial point. To distinguish between theoretical results on the estimator and possible issues due to imperfect conditional sampling, [28] presents two ways of generating conditional random variables, referred to as the Perfect and the Effective implementations. In the Perfect case, Acceptance-Rejection sampling is used to generate samples above a given threshold while in the Effective one, trajectories already following the targeted distributions are used to find an initial state $\mathbf{u}$ such that $\Phi(\mathbf{u})$ is greater or equal than the current threshold. In this example, we want to focus on the theoretical results on the number of iterations and on the estimators. Hence we make use of the Perfect sampling described in Algorithms 2.

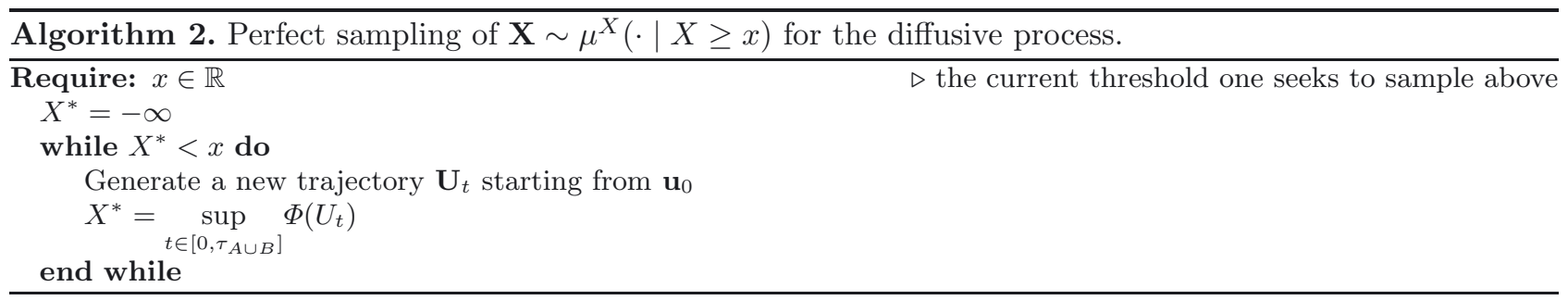

\section{Numerical results}

Here we set $\Phi(\mathbf{u})=0.5\left(1+u_{1}\right), a=0.6, b=0.3, \mathbf{u}_{0}=(-0.9,0), \beta=10$ and $\mathrm{d} t=1$ as in [28]. $\Phi(\mathbf{u})=0.5\left(1+u_{1}\right)$ and so $\Phi\left(\mathbf{u}_{0}\right)=0.05$ : with a large timestep some trajectories will go directly into $A$, producing a discontinuity in the $c d f$ of $X$. We computed reference values using a crude Monte Carlo estimator with $N=10^{6}$ and found $p=0.068$ and $\Delta=0.396$. We then set $N=300$ to get a coefficient of variation below $10 \%$ because: $\mathrm{CV}[\widehat{p}]^{2} \approx-\log p / N \Rightarrow N \approx 268$.

We first focus on the distribution of the number of iterations described in Propositions 2.6 and 2.7 and on the corrected number of iterations to get a pure Poisson's distribution (see Thm. 2.13).

Figure 3 shows the histograms of the number of iterations for the strict random walk, the non-strict one and the pure Poisson correction. Their are in good agreement with the theoretical distributions presented in equation (2.8), equation (2.7) and Theorem 2.13 respectively. Especially we can see that for a given $N$, the cost of the estimators are different. Indeed, if one considers that the cost is the number of calls to a conditional simulator, then it is equal to the final number of iterations and Figures $3 \mathrm{a}$ and $3 \mathrm{~b}$ present a clear shift: on average the discontinuity will produces $(1 / \Delta-1) N$ iterations for the non-strict random walk and only $(1-\Delta) N$ 


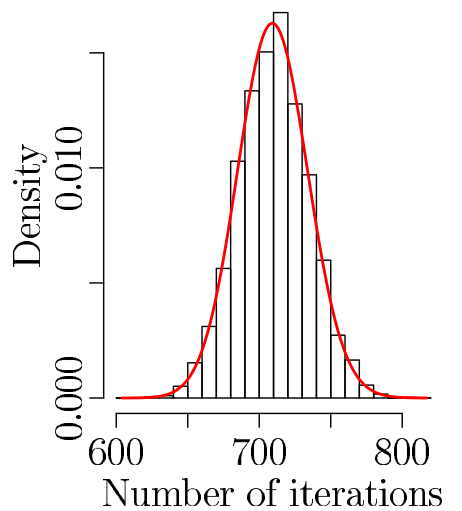

(a) Strict random walk

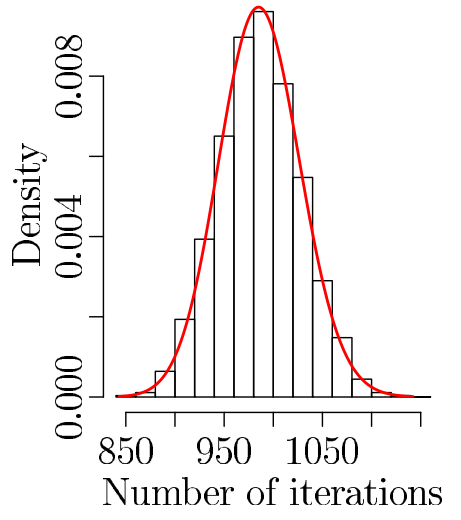

(b) Non-strict random walk

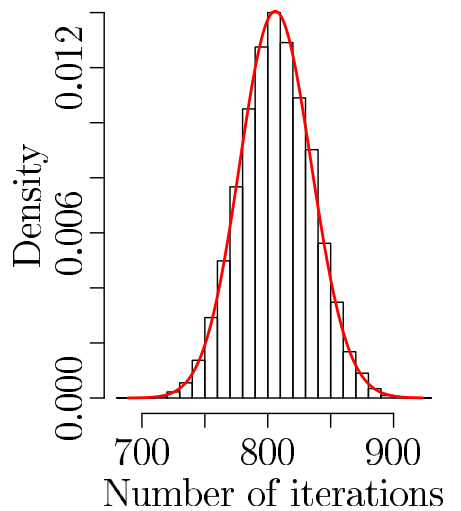

(c) Pure Poisson correction

FIGURE 3. Histogram over $10^{4}$ realisations of the number of iterations for the strict random walk (Eq. (2.8)), the non-strict one (Eq. (2.7)) and the pure Poisson correction (Thm. 2.13). $X$ has one discontinuity at $x=0.05$ and $\mathrm{P}[X>x] / \mathrm{P}[X \geq x] \approx 0.396$. The curves show the theoretical distributions.

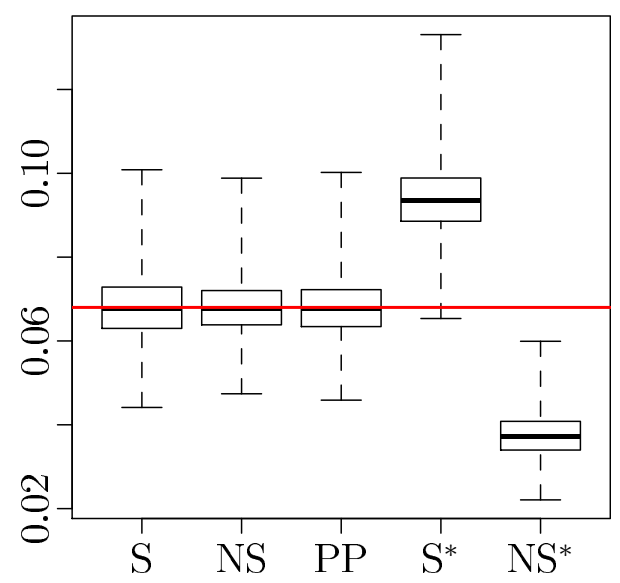

Figure 4. Boxplots of the estimation of $p \approx 0.068$ over $10^{4}$ simulations with $N=300$, whiskers extending to the extreme values. (S): strict random walk estimator (Eq. (2.14)), (NS): non-strict random walk estimator (Eq. (2.11)), (PP): pure Poisson estimator (Eq. (2.16)); with *: estimators if the discontinuity is not taken into account, i.e. $(1-1 / N)^{\text {Number of iterations }}$

for the strict random walk; with $\Delta=0.396$, this gives approximately 276 more iterations. On the histograms a shift of 250 to 300 is clearly visible in the $x$ axis.

We now check the accuracy of the probability estimators. Firstly, they should be all unbiased. Secondly, for a given $N$ one should see a variance increase from the MVUE of the non-strict random walk of equation (2.11) to the pure Poisson estimator (Eq. (2.16)) and to the MVUE of the strict random walk (Eq. (2.14)).

Figure 4 shows a boxplot of the three estimators over $10^{4}$ simulations. As an illustration, the estimators computed directly as if $X$ were continuous are also added to the plot. The horizontal line stands for the reference value calculated with a crude Monte Carlo. The estimated means are 0.068 for the strict random walk estimator, 0.068 for the non-strict one and 0.068 for the pure Poisson one. This is in good agreement with 
the estimated reference value $p=0.068$. Furthermore, the empirical variances are $5.18 \times 10^{-5}$ for the strict inequality random walk and $4.21 \times 10^{-5}$ for the pure Poisson estimator while the theoretical values given by equations 2.15 and 2.4 are $5.09 \times 10^{-5}$ and $4.16 \times 10^{-5}$. On the other hand, the probability estimators are clearly not consistent when the discontinuity is not handled properly, i.e. when the estimator is computed with the formula valid only in the continuous case (Eq. (2.2)).

All together, these numerical results are in good agreement with the the theoretical ones.

\subsection{Discrete random variable}

Counting problems are typical cases where the random variable of interest is known to be integer-valued. Indeed, it is shown that many of these problems can be put into the setting of estimating extreme probability $[4,5,21,22]$. Among other, we focus here on the Boolean SATisfiability Problem (SAT problem). We do not pretend being competitive against specific $S A T$ solvers. Instead, we use this test case because the random variable will have several discontinuity points not only at the origin.

\section{The SAT problem}

A SAT problem comprises 1) a binary vector of $n$ literals which can be either TRUE $(=1)$ of FALSE $(=0)$ : $\mathbf{u}=\left(u_{1}, \ldots, u_{n}\right)$ is called a truth assignment, e.g. $\mathbf{u}=(\operatorname{TRUE}, \mathrm{TRUE}, \ldots, \mathrm{FALSE})=(1,1, \ldots, 0)$ and 2$)$ a set of $m$ clauses $\left\{S_{1}, \ldots, S_{m}\right\}$ expressed as OR logical operators (also denoted by $\vee$ ) on the literals, e.g. $S_{i}=u_{i_{1}} \vee u_{i_{2}} \vee \ldots \vee u_{i_{k}}$.

The SAT problem in itself is then defined as follows: find an assignment $\mathbf{u}$ such that all clauses are true $(S A T$ assignment problem) or count the number of different assignments which satisfy all the clauses (\#SAT). The conjunctive normal form $(\mathrm{CNF})$ of a SAT problem is then the product (logical operator AND or $\wedge$ ) of all clauses $F=S_{1} \wedge \ldots \wedge S_{m}$ and both problems can be rewritten:

SAT assignment problem: is there at least one $\mathbf{u} \in\{0,1\}^{n}$ such that $F(\mathbf{u})=$ TRUE ?

\#SAT: find $\operatorname{card}(\mathcal{S})=|\mathcal{S}|$ with $\mathcal{S}=\left\{\mathbf{u} \in\{0,1\}^{n} \mid F(\mathbf{u})=\right.$ TRUE $\}$

If one considers $\mathbf{U}$ a Uniform random vector on $\{0,1\}^{n}$, then it is known [27] that:

$$
p_{m}=\mathrm{P}[\mathbf{U} \in \mathcal{S}]=\frac{|\mathcal{S}|}{2^{n}} .
$$

Hence one can build an estimator of $|\mathcal{S}|$ by estimating the (extreme) probability $p_{m}$. In order to make use of the results of Section 2.3, one can consider the discrete random variable $X=S(\mathbf{U})$ of the number of clauses satisfied by the assignment $\mathbf{U}$ :

$$
X=S(\mathbf{U})=\sum_{i=1}^{m} S_{i}(\mathbf{U})
$$

Therefore $X \in \llbracket 0, m \rrbracket$ and:

$$
\mathrm{P}[\mathbf{U} \in \mathcal{S}]=\mathrm{P}[F(\mathbf{U})=\mathrm{TRUE}]=\mathrm{P}[X=m]=\mathrm{P}[X \geq m] .
$$

\section{Conditional simulations}

In order to perform the conditional simulations $\mu^{X}(\cdot \mid X \geq i)$ to simulate the random walks, we propose to use the Gibbs sampler as described in [24] or [10]. Indeed, $\mu^{X}(\cdot \mid X \geq i)=\mu^{U}(\cdot \mid S(\mathbf{U}) \geq i)$. Starting from a sample $\mathbf{U}^{*}$ such that $S\left(\mathbf{U}^{*}\right) \geq i$, it resamples each coordinate in a deterministic (systematic Gibbs sampler) or randomized order conditionally to the other ones to stay in the right domain. A sketch of a Gibbs sampler is given in Algorithm 3. Practically speaking, to avoid local maxima and improve the convergence of the Markov chain, we do not start from the current $\mathbf{U}$ such that $X_{i}=S(\mathbf{U})$. Instead, we pick at random a starting point $\mathbf{U}^{*}$ in a population already following the target distribution, i.e. such that $S\left(\mathbf{U}^{*}\right) \geq X_{i}$. This population is built on-the-fly with all the generated samples $\mathbf{U}$ such that $S(\mathbf{U}) \geq X_{i}$. Especially each fail of a Geometric law 


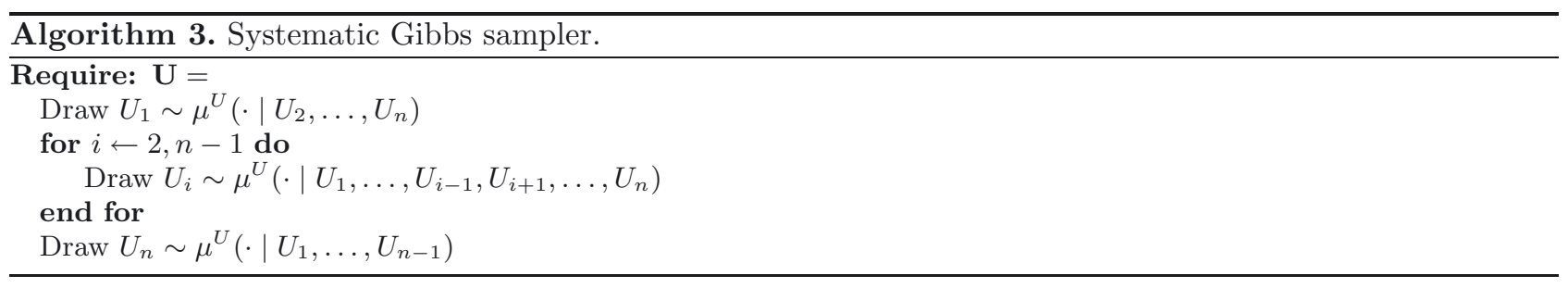

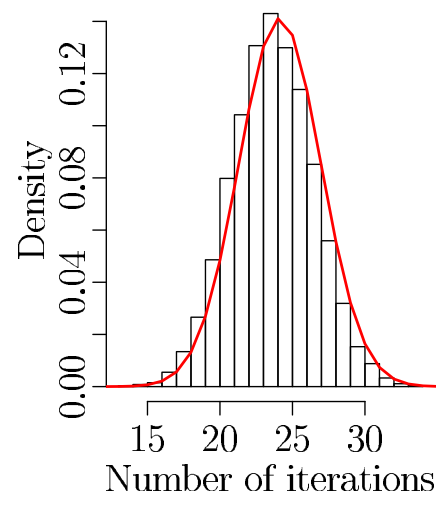

(a) Strict random walk

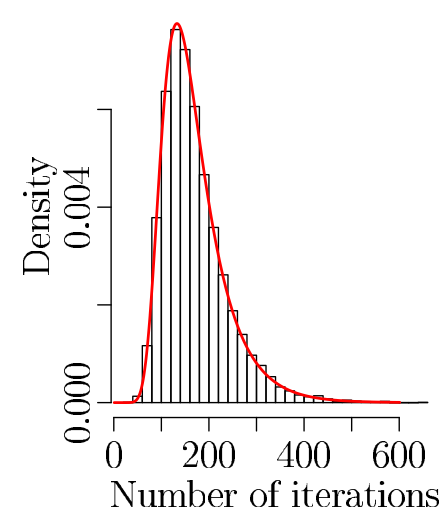

(b) Non-strict random walk

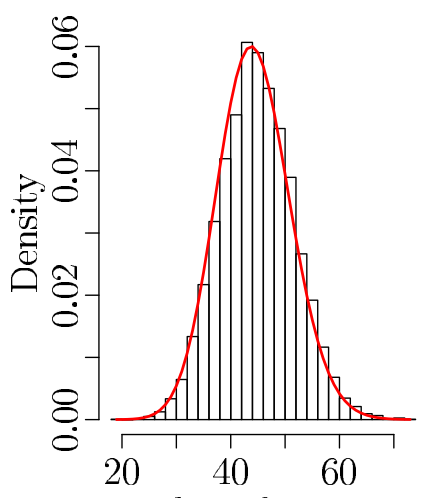

Number of iterations

(c) Pure Poisson correction

FiguRE 5. Histogram over $10^{4}$ realisations of the number of iterations for the strict random walk (Eq. (2.8)), the non-strict one (Eq. (2.7)) and the pure Poisson correction (Thm. 2.13). $X$ is an integer-valued random variable with up to 325 jump points. The curves show the theoretical distributions with estimated Geometric parameters $\Delta_{d}$ with $N=50000$.

will increase its size instead of replacing the previous vector as it is usual in a Multilevel Splitting method. We point out the fact that we focus on the real-valued random variable of the number of satisfied clauses and that multidimensional vectors are only seen as a way to generate it and to make conditional sampling on it. This makes us simulating the random walks by batches of size $k$ on the model of [30]: at a given iteration only the smallest states are moved on. Reader is referred to [30] for further details on parallel implementation.

Finally, we also generate both the strict and the non-strict random walks in the same run. This is to focus on the statistical properties of the number of iterations and of the estimators. Here some $\Delta_{d}=\mathrm{P}[X>d] / \mathrm{P}[X \geq d]$ are very small, so that the size of the population for the conditional sampling may become very small if one only keeps those starting points strictly above the current threshold.

\section{Numerical results}

We consider here the $S A T$ problem referred to as uf75-01 on satlib.org, also used by [6], who provide a reference value $p=5.977 \times 10^{-20}$ with a relative error of $0.03 \%$. It has $m=325$ clauses in dimension $n=75$. Hence $X$ is a discrete random variable with up to 325 jump points. We first focus on the number of iterations of the random walks. To do so, we simulate $N=10^{4}$ random walks as well as the pure Poisson correction. Figure 5 plots the histograms of the random number of iterations for each case and theoretical curves with the $\Delta_{d}$ estimated using an other simulation with $N=50000$.

These plots show a good consistency between theoretical formulae (Eqs. (2.7) and (2.8) and Thm. (2.13)) and numerical results. Especially with a lot of jump points, the number of iterations are very different from Figure 5a to Figure 5b (almost hundred times bigger). 


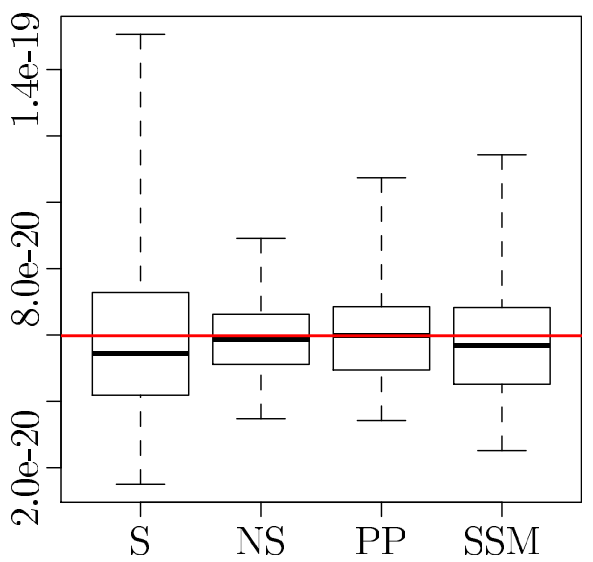

FIGURE 6 . Boxplots of the estimation of $p \approx 5.977 \times 10^{-20}$ over 100 simulations with $N=1000$, whiskers extending to the extreme values. (S): Strict random walk estimator (Eq. (2.14)), (NS): Non-strict random walk estimator (Eq. (2.11)), (PP): Pure Poisson estimator (Eq. (2.16)), (SSM): Smoothed Splitting Method [10] with $N_{\mathrm{SSM}}=8000$.

We now focus on the probability estimators. In this scope we also consider the Smoothed Splitting Method $(S S M)$ [10], which uses a case-specific continuous auxiliary random variable. The aim of this benchmark is to assess the relevance of using such transformations instead of considering the original random variable with the MVUE we have proposed in Section 2.3. We refer the reader to [10] for further details on this transformation. The algorithm is then a usual Multilevel Splitting method with $p_{0}=0.2$. We set $N_{\mathrm{SSM}}$ such that the total numbers of simulated samples for the non-strict random walk and for the SSM are of the same order of magnitude. The non-strict random walk generates on average 169.683 samples while an AMS with $p_{0}=0.2$ typically generates $\left(1-p_{0}\right) N \log p / \log p_{0}$ samples. We set $N=1000$ for the random walks, which gives $N_{\mathrm{SSM}} \approx 7712$. Here we set $N_{\mathrm{SSM}}=8000$.

With a lot of discontinuity points, the variance differences between the three estimators is clearer, especially between the non-strict random walk estimator and the pure Poisson one. Also the strict inequality estimator has a much bigger variance. Indeed, for some discontinuity points $d \in D_{x}, \Delta_{d} \approx 10^{-2}$ while $N=1000$, which gives coefficients of variations around $1 / \sqrt{N p} \approx 32 \%$. This is not an issue in the non-strict case as the coefficient of variation of the MVUE of Lemma 2.8 typically scales like $\sqrt{(1-p) / N}$. On the other hand, over the 100 simulations, we have an estimation of $\operatorname{card}\left(D_{x}\right) \approx 66$, which gives an upper bound for the squared coefficient of variation in the non-strict case (see Eq. (2.13)): 0.068, while the estimated squared coefficient of variation is 0.032 . Concerning the $S S M$ estimator, we have found a coefficient of variation of 0.33 while the theoretical value should be 0.117 . As already noticed by [10] this is due to a non-perfect implementation of the Multilevel Splitting. This limitation is less visible while keeping the discrete random variable because we save all generated samples and so improve the approximation of the target distribution at each iteration.

Finally, these numerical results with a discrete random variable show a good consistency with the theoretical ones. Also it appears that it may not be relevant to transform the problem to consider a Multilevel Splitting method on a continuous random variable because this can make the conditional simulations harder to approximate. The pure Poisson correction is in this context a good trade-off between accuracy and knowledge of the distribution. Furthermore, it is just a by-product of the non-strict random walk and so the MVUE can also be computed in the same run. Concerning the strict inequality random walk, it may suffer from two limitations if the some $\Delta_{d}$ are very small (typically if $1 / \Delta_{d}$ becomes of the order of the total population size $N$ ) because 1) the coefficient of variation of the MVUE of Lemma 2.9 is $\approx \sqrt{1 /\left(N \Delta_{d}\right)}$, and 2 ) Markov chain drawing may be poor because only few samples will be available in the right domain: at each iteration, only the samples 
strictly above the current threshold are kept, so on average only $N \Delta_{d}$ samples will be in the right domain. If Markov chain drawing is used to approximate conditional sampling, then the diversity of the population may decay strongly iterations after iterations.

\section{Conclusion}

The goal of this paper was to study the impact of using Multilevel Splitting methods on potentially discontinuous random variables, especially the parallel optimal (minimal variance) adaptive Multilevel Splitting method, also called the Last Particle Algorithm which only replaces the smallest particle at a given iteration. This implementation had been shown to be a particular case of generating several point processes associated with a real-valued random variable, those point processes, also called increasing random walk here (see Sect. 2.1) being related to a Poisson process with parameter 1 . To handle potential discontinuities, we had to distinguish between strict and non-strict inequality for conditional sampling. Following [28] who gave the law of the number of iterations of such algorithms with non-strict inequality, we have been able to extend this result to the strict inequality random walk. Furthermore, we have given the Minimal Variance Unbiased Estimator of the probability of exceeding a threshold in both cases. These estimators eventually become the same when the random variable of interest is actually continuous. On the other hand their distributions are not easy to characterise. In this scope, we have proposed a third estimator based on the non-strict inequality implementation. It has always the same statistical properties, $X$ being continuous or not, precisely the properties for continuous random variables recalled in Section 2.1. These properties are well known and allow for building confidence intervals on the estimator. All these theoretical results have been challenged on two examples and proved good agreement with the practical results.

Practically speaking, the strict inequality implementation may be less efficient if conditional sampling has to be done with Markov chain drawing and can even return 0 if some jump points $d \in D_{x}$ are such that $\Delta_{d}^{-1}=\mathrm{P}[X \geq d] / \mathrm{P}[X>d]$ is of the order of the population size: this is due to the estimation of the $\Delta_{d}$ with crude Monte Carlo. The non-strict implementation prevents from such issues because it generates Geometric random variables. On the other hand there is no literal expression of the variance of its estimator. At the cost of generating independent Uniform random variables, the non-strict implementation can also output the pure Poisson estimator, it is the estimator with known distribution which does not depend on the discontinuities of $X$. If one only want to focus on the output without worrying about discontinuities, this latter appears as good trade-off.

Finally, considering the global cost of an estimator against its variance, some optimisation may be done to start some random walks only from a given point and/or stop them before the targeted value because all the jumps are not of equal size. This has not been studied here and is let for further research.

\section{APPENDIX}

Proof of Proposition 2.6. The distribution of $M_{x}$ has already been shown in [28] assuming that card $D_{x}<\infty$. We extend this result to the possibly countably infinite number of discontinuities.

Let $S_{n}=\{x \in \mathbb{R} \mid 0<\mathrm{P}[X=x]<1 / n\}$ be the set of the jump points of $F_{X}$ with jump amplitudes smaller than $1 / n$ and $X^{(n)}=X \mathbb{1}_{X \notin S_{n}}$. $X^{(n)}$ has a finite number of jump points and accumulates the (possibly infinite) number of jump points $d \in D \mid \mathrm{P}[X=d]<1 / n$ at 0 . Since it has a finite number of discontinuities, the law of its associated counting random variable is then known.

Furthermore it verifies:

$$
\forall x \in \mathbb{R}, \mathrm{P}\left[X^{(n)} \leq x\right]=\mathrm{P}[X \leq x]+\mathbb{1}_{x \geq 0} \sum_{\substack{d \in S_{n} \\ d>x}} \mathrm{P}[X=d]-\mathbb{1}_{x<0} \sum_{\substack{d \in S_{n} \\ d \leq x}} \mathrm{P}[X=d] .
$$


Hence $X^{(n)} \underset{n \rightarrow \infty}{\stackrel{\mathcal{L}}{\longrightarrow}} X$, which implies $M_{x}^{(n)} \underset{n \rightarrow \infty}{\stackrel{\mathcal{L}}{\longrightarrow}} M_{x}$ with $M_{x}^{(n)}$ the counting random variable at state $x$ associated with an increasing random walk on $X^{(n)}$.

$$
\begin{gathered}
\text { Moreover, one has: } \forall x \in \mathbb{R}, \forall n \geq 1, M_{x}^{(n)} \sim \mathcal{P}\left(-\log \frac{\mathrm{P}\left[X^{(n)}>x\right]}{\prod_{d \in D_{x} \backslash S_{n}} \Delta_{d}}\right)+\sum_{d \in D_{x} \backslash S_{n}} \mathcal{G}\left(\Delta_{d}\right) \text {. Finally, this gives: } \\
M_{x} \sim \mathcal{P}\left(-\log \frac{\mathrm{P}[X>x]}{\prod_{d \in D_{x}} \Delta_{d}}\right)+\sum_{d \in D_{x}} \mathcal{G}\left(\Delta_{d}\right) .
\end{gathered}
$$

We also show that the first and second order moments of $M_{x}$ remain finite even when $\operatorname{card}\left(D_{x}\right)=\infty$. One has:

$$
0 \leq \sum_{d \in D_{x}}\left(\frac{1}{\Delta_{d}}-1\right)=\sum_{d \in D_{x}} \frac{\mathrm{P}[X=d]}{\mathrm{P}[X>d]} \leq \frac{1}{\mathrm{P}[X>x]} \sum_{d \in D_{x}} \mathrm{P}[X=d] \leq \frac{1-p_{x}}{p_{x}}
$$

and:

$$
1 \geq \prod_{d \in D_{x}} \Delta_{d} \geq \prod_{d \in D_{x}} \mathrm{e}^{-\left(\frac{1}{\Delta_{d}}-1\right)} \geq \mathrm{e}^{-\sum_{d \in D_{x}}\left(\frac{1}{\Delta_{D}}-1\right)} \geq \mathrm{e}^{-\frac{1-p_{x}}{p_{x}}}>0 .
$$

All together, theses inequalities give the result:

$$
\begin{gathered}
\mathrm{E}\left[M_{x}\right]=-\log \frac{p_{x}}{\prod_{d \in D_{x}} \Delta}+\sum_{d \in D_{x}}^{N}\left(\frac{1}{\Delta_{d}}-1\right) \leq-\log p_{x}+\frac{1-p_{x}}{p_{x}} \\
\operatorname{var}\left[M_{x}\right]=-\log \frac{p_{x}}{\prod_{d \in D_{x}} \Delta}+\sum_{d \in D_{x}}^{N} \frac{1}{\Delta_{d}}\left(\frac{1}{\Delta_{d}}-1\right) \leq-\log p_{x}+\frac{1-p_{x}}{p_{x}^{2}} .
\end{gathered}
$$

Proof of Proposition 2.7. Using the renewal property of the Poisson's Process the number of events in the continuous part will be the same as the one in the non-strict case. Indeed the only difference with the non-strict random walk comes from the behaviour of the random walk when $X_{n} \in D_{x}$. In this latter case, while the non-strict inequality repeats the trial until success, the strict inequality do it only once. Hence the Geometric law is replaced by a Bernoulli one.

Proof of Lemma 2.8. One is going to use Lehmann-Scheffé's theorem with the statistic $T=\sum_{i=1}^{N} G_{i}$. As the sum of $N$ independent Geometric random variables with parameter $p, T$ follows a Negative Binomial law: $\forall t \in \mathbb{N}, \mathrm{P}[T=t]=\left(\begin{array}{c}N+t-1 \\ t\end{array}\right) p^{N}(1-p)^{t} . T$ is sufficient:

$$
\mathcal{L}\left(g_{1}, \ldots, g_{N}, p\right)=\prod_{i=1}^{N} \mathrm{P}\left[G_{i}=g_{i}\right]=(1-p)^{\sum_{i=1}^{N} g_{i}} p^{N}=(1-p)^{t} p^{N} .
$$

$T$ is also complete: let $\phi$ be a measurable function, one has:

$$
\begin{aligned}
\forall p \in(0,1), \mathrm{E}[\phi(T)]=0 & \Rightarrow \forall p \in(0,1), p^{N} \sum_{t=0}^{\infty}\left(\begin{array}{c}
t+N-1 \\
t
\end{array}\right) \phi(t)(1-p)^{t}=0 \\
& \Rightarrow \forall \theta \in(0,1), \sum_{t=0}^{\infty} \alpha_{t} \theta^{t}
\end{aligned}
$$


with $\alpha_{t}=\left(\begin{array}{c}t+N-1 \\ t\end{array}\right) \phi(t)$ and $\theta=1-p$. Furthermore $p=1$ i.e. $\theta=0$ gives $\phi(0)=0$ and $\theta=1$, i.e. $p=0$ gives $\mathrm{P}[T<\infty]=0$. Hence the power series $\theta \mapsto \sum \alpha_{t} \theta^{t}$ is identically null on its radius of convergence $[0,1)$ and so $\forall t \in \mathbb{N}, \alpha_{t}=0$, which means $\forall t \in \mathbb{N}, \phi(t)=0$ and $T$ is complete.

We now consider the estimator $R=\frac{1}{N} \sum_{i=1}^{N} \mathbb{1}_{G_{i}=0} . R$ is unbiased because $\mathrm{E}\left[\mathbb{1}_{G_{i}=0}\right]=\mathrm{P}\left[G_{1}=0\right]=p$. Then Lehmann-Scheffé's theorem states that $\mathrm{E}[R \mid T]$ is the MVUE of $p$. This gives:

$$
\begin{aligned}
\mathrm{E}[R \mid T=t] & =\frac{1}{N} \sum_{i=1}^{N} \mathrm{E}\left[\mathbb{1}_{G_{i}=0} \mid \sum_{i=1}^{N} G_{i}=t\right]=\mathrm{P}\left[G_{1}=0 \mid \sum_{i=1}^{N} G_{i}=t\right] \\
& =\frac{\mathrm{P}\left[G_{1}=0, \sum_{i=2}^{N} G_{i}=t\right]}{\mathrm{P}\left[\sum_{i=1}^{N} G_{i}=t\right]}=\mathrm{P}\left[G_{1}=0\right] \frac{\left(\begin{array}{c}
t+N-2 \\
t
\end{array}\right) p^{N-1}(1-p)^{t}}{\left(\begin{array}{c}
t+N-1 \\
t
\end{array}\right) p^{N}(1-p)^{t}} \\
\mathrm{E}[R \mid T=t] & =\frac{N-1}{N-1+t} .
\end{aligned}
$$

Hence, $\widehat{p}=\mathrm{E}[R \mid T]=(N-1) /(N-1+T)$ is the MVUE of $p$.

Proof of Proposition 2.11. On the one hand, for all $a<b$ such that $X$ is continuous on $(a, b), \mathrm{P}[X>b \mid X>a]$ can be estimated by $(1-1 / N) \#\left\{X_{n} \in(a, b)\right\}$ with $\#\left\{X_{n} \in(a, b)\right\}$ the number of events of the sum process in $(a, b)$. Moreover, since $b \mapsto \mathrm{P}[X \geq b]$ is left-continuous, it is also an MVUE of $\mathrm{P}[X \geq b \mid X>a]$ and this relation remains true if $b \rightarrow a$ since $\operatorname{card}(\varnothing)=0$. Using the fact that the RLE of $\left(X_{1}, \ldots, X_{M_{x}}\right), \mathbf{r}=(1, \ldots, 1)$ with probability 1 when $X$ is continuous, $(1-1 / N)^{\#\left\{X_{n} \in(a, b)\right\}}=\prod(N-1) /\left(N-1+r_{i}\right)$ with probability 1.

On the other hand $\forall d \in D_{x}, \Delta_{d}=\mathrm{P}[X>d] / \mathrm{P}[X \geq d]$ can be estimated with the MVUE defined in Lemma 2.8. The first state of each chain non smaller than $d$ can be considered as an iid. sample of $X \mid X \geq d$. The number of times $d$ is found in $\left(X_{1}, \ldots, X_{M_{x}}\right)$ is then the sum of $N$ realisations of a Geometric random variable with parameter $\Delta_{d}$. Hence, $\Delta_{d}$ is estimated with $(N-1) /\left(N-1+r_{d}\right)$ with $r_{d}$ the number of times $d$ is found in $\left(X_{1}, \ldots, X_{M_{x}}\right)$.

Since $D$ is countable, we consider $\mathbb{R} \backslash D=\bigcup_{i} I_{i}$ with $\left(I_{i}\right)_{i}$ a sequence of disjoint open intervals. Note that some subsequence of $I_{n}$ may converge toward the empty set if $D$ is infinite countable with some accumulation points. However $X$ is continuous on $\mathbb{R} \backslash D$. Using the renewal property of the Poisson process, one can consider that $\prod(N-1) /\left(N-1+r_{i}\right)$ is a product of independent MVUE estimators. Especially, denoting by $M_{\text {pois }}$ the number of 1 in $\mathbf{r},(1-1 / N)^{M_{\text {pois }}}$ is a MVUE of $p_{\text {pois }}:=p_{x} / \prod \Delta_{d}$.

We now explicit the calculation for the bounds on the variance. One has:

$$
\mathrm{E}\left[\widehat{p}_{x}^{2}\right]=p_{\text {pois }}^{2} p_{\text {pois }}^{-1 / N} \prod_{d \in D_{x}} \mathrm{E}\left[\left(\frac{N-1}{N-1+T_{d}}\right)^{2}\right]
$$

with $T_{d} \sim \operatorname{Neg} \operatorname{Bin}\left(N, \Delta_{d}\right)$. For a given $d \in D_{x}$, one has:

$$
\begin{aligned}
\mathrm{E}\left[\left(\frac{N-1}{N-1+T_{d}}\right)^{2}\right] & =\sum_{t=0}^{\infty}\left(\begin{array}{c}
t+N-1 \\
t
\end{array}\right)\left(1-\Delta_{d}\right)^{t} \Delta_{d}^{N}\left(\frac{N-1}{N-1+t}\right)^{2} \\
& =\sum_{t=0}^{\infty}\left(\begin{array}{c}
t+N-2 \\
t
\end{array}\right)\left(1-\Delta_{d}\right)^{t} \Delta_{d}^{N} \frac{N-1}{N-1+t} \\
& =\Delta_{d} \sum_{t=0}^{\infty}\left(\begin{array}{c}
t+N-2 \\
t
\end{array}\right)\left(1-\Delta_{d}\right)^{t} \Delta_{d}^{N-1} \frac{N-2}{N-2+t} \frac{N-1}{N-2} \frac{N-2+t}{N-1+t}
\end{aligned}
$$


Furthermore, $\forall t \geq 0, \frac{N-1}{N-2} \frac{N-2+t}{N-1+t} \in[1,(N-1) /(N-2)]$, which gives:

$$
\Delta_{d}^{2} \leq \mathrm{E}\left[\left(\frac{N-1}{N-1+T_{d}}\right)^{2}\right] \leq \Delta_{d}^{2} \frac{N-1}{N-2}
$$

Eventually the variance writes:

$$
p_{x}^{2}\left(p_{\text {pois }}^{-1 / N}-1\right) \leq \operatorname{var}\left[\widehat{p}_{x}\right]=\mathrm{E}\left[\widehat{p}_{x}^{2}\right]-p_{x}^{2} \leq p_{x}^{2}\left(p_{\text {pois }}^{-1 / N} \prod_{d \in D_{x}} \frac{N-1}{N-2}-1\right) .
$$

Proof of Proposition 2.12. The same reasoning as for the Proof of Proposition 2.11 applies where the MVUE of a Geometric law is replaced by the one of a Bernoulli distribution. For the variance one has:

$$
\begin{aligned}
& \mathrm{E}\left[\widehat{p}_{x}^{2}\right]=p_{\text {pois }}^{2} p_{\text {pois }}^{-1 / N} \prod_{d \in D_{x}} \frac{\Delta_{d}^{2}}{N}\left(N-1+\frac{1}{\Delta_{d}}\right) \\
& \mathrm{E}\left[\hat{p}_{x}^{2}\right]=p_{x}^{2} p_{x}^{-1 / N} \prod_{d \in D_{x}} \frac{\Delta_{d}(N-1)+1}{N \Delta_{d}^{1-1 / N}}
\end{aligned}
$$

which gives the result.

Proof of Theorem 2.13. The difference between the generation of the non-strict random walk and Algorithm 1 stands in the addition of the while loop from line 6 to line 12. This loop is entered when a Geometric scheme is started: two consecutive events being equal is a non-zero probability event only when $X_{n} \in D$. In this context, the condition $\left\{U_{n+1}>U_{n}\right\}$ lets generate the counting random variable of the continuous random variable associated with the Geometric scheme as explained in Section 2.3. Therefore it follows a Poisson distribution with parameter $-\log \Delta_{X_{n}}$. The renewal properties of the Poisson Process lets conclude the proof.

Acknowledgements. The author would like to thank his advisors Josselin Garnier (University Paris Diderot) and Gilles Defaux (Commissariat à l'Énergie Atomique et aux Énergies Alternatives) for their advice and suggestions as well as the referee and the editor for their clear and helpful comments.

\section{REFERENCES}

[1] M. Amrein and H.R. Künsch, A variant of importance splitting for rare event estimation: Fixed number of successes. $A C M$ Trans. Model. Comput. Simul. (TOMACS) (2011).

[2] S.-K. Au and J.L. Beck, Estimation of small failure probabilities in high dimensions by subset simulation. Probab. Eng. Mech. 16 (2001) 263-277.

[3] A. Beskos, A. Jasra, N. Kantas and A. Thiery, On the convergence of adaptive sequential Monte Carlo methods. To appear in Ann. Appl. Probab. (2015).

[4] I. Bezáková, D. Štefankovic, V.V. Vazirani and E. Vigoda, Accelerating simulated annealing for the permanent and combinatorial counting problems. SIAM J. Comput. 37 (2008) 1429-1454.

[5] Z.I. Botev and D.P. Kroese, An efficient algorithm for rare-event probability estimation, combinatorial optimization, and counting. Methodology Comput. Appl. Probab. 10 (2008) 471-505.

[6] Z.I. Botev and D.P. Kroese, Efficient Monte Carlo simulation via the generalized splitting method. Stat. Comput. 22 (2012) $1-16$.

[7] C.-E. Brérhier, T. Leliévre and M. Rousset, Analysis of adaptive multilevel splitting algorithms in an idealized case. ESAIM: PS 19 (2015) 361-394.

[8] F. Cerou, P. Del Moral, A. Guyader and F. Malrieu, Fluctuation analysis of adaptive multilevel splitting. Preprint arXiv: $1408.6366(2014)$.

[9] F. Cérou and A. Guyader, Adaptive multilevel splitting for rare event analysis. Stoch. Anal. Appl. 25 (2007) $417-443$.

[10] F. Cérou, A. Guyader, R. Rubinstein and R. Vaisman, On the use of smoothing to improve the performance of the splitting method. Stochastic Models 27 (2011) 629-650. 
[11] F. Cérou, P. Del Moral, T. Furon and A. Guyader, Sequential Monte Carlo for rare event estimation. Stat. Comput. 22 (2012) 795-808.

[12] B. Charles-Edouard, G. Maxime, G. Ludovic, L. Tony and R. Mathias, Unbiasedness of some generalized adaptive multilevel splitting algorithms. e-prints Preprint arXiv:1505.02674 (2015).

[13] P. Del Moral, A. Doucet and A. Jasra, Sequential Monte Carlo samplers. J. Roy. Statist. Soc.: Ser. B (Statistical Methodology) 68 (2006) 411-436.

[14] A. Froda, Sur la Distribution des Propriétés de Voisinage des Fonctions de Variables Réelles. Ph.D. thesis, Université de Paris (1929).

[15] M.J.J. Garvels, The splitting method in rare event simulation. Universiteit Twente (2000).

[16] P. Glasserman, P. Heidelberger, P. Shahabuddin and T. Zajic, Multilevel splitting for estimating rare event probabilities. Oper. Res. 4 (1999) 585-600.

[17] A. Guyader, N. Hengartner and E. Matzner-Løber, Simulation and estimation of extreme quantiles and extreme probabilities. Appl. Math. Optim. 64 (2011) 171-196.

[18] H. Hoos and T. Stiitzle, SATLlB: An online resource for research on SAT. Sat2000: highlights of satisfiability research in the year 2000 (2000) 283.

[19] M. Huber and S. Schott, Using TPA for Bayesian Inference. Bayesian Statistics 9 (2011) 257-282.

[20] H. Kahn and T.E. Harris, Estimation of Particle Transmission by Random Sampling. National Bureau of Standards Applied Mathematics Series 12 (1951) 27-30.

[21] M. Mitzenmacher and E. Upfal, Probability and Computing: Randomized Algorithms and Probabilistic Analysis. Cambridge University Press (2005).

[22] R. Motwani and P. Raghavan, Randomized Algorithms. Chapman \& Hall/CRC (2010).

[23] M. Rosenbluth and A. Rosenbluth, Monte Carlo calculation of the average extension of molecular chains. J. Chem. Phys. 23 (2004) 356-359.

[24] R. Rubinstein, The Gibbs cloner for combinatorial optimization, counting and sampling. Methodol. Comput. Appl. Probab. 11 (2009) 491-549.

[25] R. Rubinstein, Randomized algorithms with splitting: Why the classic randomized algorithms do not work and how to make them work. Methodol. Comput. Appl. Probab. 12 (2010) 1-50.

[26] R. Rubinstein, A. Dolgin and R. Vaisman, The splitting method for decision making. Commun. Statistics-Simul. Comput. 41 (2012) 905-921.

[27] R.Y. Rubinstein and D.P. Kroese, Simulation and the Monte Carlo method. In vol. 707. John Wiley \& Sons (2011).

[28] E. Simonnet, Combinatorial analysis of the adaptive last particle method. To appear in Stat. Comput. (2014). Doi: $10.1007 / \mathrm{s} 11222-014-9489-6$.

[29] J. Skilling, Nested sampling for general bayesian computation. Bayesian Analysis 1 (2006) 833-859.

[30] C. Walter, Moving particles: A parallel optimal multilevel splitting method with application in quantiles estimation and meta-model based algorithms. Structural Safety 55 (2015) 10-25.

[31] C. Walter, Point process-based Monte Carlo estimation. To appear in Stat. Comput. (2015) Doi: 10.1007/s11222-015-9617-y.

[32] C. Walter and G. Defaux, Rare event simulation: a point process interpretation with application in probability and quantile estimation. Proc. of the 12th International Conference on Applications of Statistics and Probability (2015). 